\title{
Staging, nomograms and other predictive tools in retroperitoneal soft tissue sarcoma
}

\author{
Hannah L. Tattersall ${ }^{1}$, Dario Callegaro ${ }^{2}$, Samuel J. Ford ${ }^{1}$, Alessandro Gronchi ${ }^{2}$ \\ ${ }^{1}$ Midlands Abdominal and Retroperitoneal Sarcoma Unit (MARSU), Queen Elizabeth Hospital, Birmingham, UK; ${ }^{2}$ Department of Surgery, \\ Fondazione IRCCS Istituto Nazionale dei Tumori, Milan, Italy \\ Contributions: (I) Conception and design: All authors; (II) Administrative support: All authors; (III) Provision of study materials or patients: None; \\ (IV) Collection and assembly of data: None; (V) Data analysis and interpretation: None; (VI) Manuscript writing: All authors; (VII) Final approval of \\ manuscript: All authors. \\ Correspondence to: Alessandro Gronchi, MD. Department of Surgery, Fondazione IRCCS Istituto Nazionale dei Tumori, via Venezian 1, 20133 Milan, \\ Italy. Email: alessandro.gronchi@istitutotumori.mi.it.
}

\begin{abstract}
Patient outcomes following surgical resection of retroperitoneal sarcomas (RPSs) are variable and therefore predicting prognosis is challenging. The risk of recurrence varies according to several patienttumour- and treatment-related characteristics, including histological subtype and tumour grade. In an era of increased movement towards individualised patient care, the ability to predict prognosis following surgery for RPS is essential. The accurate prediction of an individual patient's outcome is important to allow adequate patient counselling and to ensure the optimal therapeutic strategy is selected. The outcomes of patients who have undergone resection for RPS can be predicted using tools such as nomograms. Nomograms take into account the relative contribution of each variable by giving them different weights and then combining them. This review aims to discuss current staging systems available for RPS and to critically appraise predictive tools that are currently available for use in clinical practice.
\end{abstract}

Keywords: Sarcoma; retroperitoneal sarcoma (RPS); nomogram; prognosis; outcome

Submitted Jul 16, 2018. Accepted for publication Jul 25, 2018.

doi: $10.21037 /$ cco.2018.08.01

View this article at: http://dx.doi.org/10.21037/cco.2018.08.01

\section{Introduction}

\section{Predicting prognosis in retroperitoneal sarcomas (RPS)}

RPSs are rare tumours accounting for approximately $0.15 \%$ of malignancies (1). Surgery to achieve local control is the only potentially curative treatment for primary localised disease and should be carried out in high volume, specialised centres $(2,3)$. The 10-year overall survival (OS) in patients with primary resected RPS is around $46 \%$, however this varies according to histological subtype (4).

In total, there are more than 60 different histological subtypes of soft tissue sarcoma (STS). Despite the large number of histological variants, 5 main histological subtypes account for $90 \%$ of RPSs $(4,5)$. Each subtype is associated with a variable prognosis due to different risks of local and/ or distant recurrence and a difference in the time taken for such events to occur. The 5 main histological subtypes seen in RPS are well-differentiated liposarcoma (WDLPS), dedifferentiated liposarcoma (DDLPS), leiomyosarcoma (LMS), solitary fibrous tumour (SFT) and malignant peripheral nerve sheath tumours (MPNST) (4).

WDLPS and SFTs tend to have more favourable outcomes with a 7-year OS of over $80 \%$ (4). Liposarcomas are the commonest histology found in the retroperitoneum comprising $50 \%$ of tumours, WDLPS account for $25 \%$ of these (4). WDLPS are low grade tumours that often recur locally several years after surgery and therefore require long-term follow up $(4,6,7)$.

SFTs account for $6 \%$ of all RPSs (4). More than $90 \%$ of these tumours are cured by surgical resection, however a small minority are particularly aggressive and can metastasise. Local recurrence is rare with a rate of $10 \%$ at 
7 years $(4,6,8)$.

DDLPS, LMS and MPNST are considered to be high-risk histological subtypes. DDLPS can be subdivided according to whether they are intermediate or high grade. Intermediate grade DDLPS tends to recur locally (40\% at 7 years), but the risk of metastasis is low ( $10 \%$ at 7 years) (4). High grade DDLPS has the same risk of local recurrence, but a much higher risk of distant metastasis (30\% at 7 years) (4). Intermediate grade DDLPS and high grade DDLPS have a 7 -year OS of $50 \%$ and $30 \%$, respectively (4).

LMS represent $20 \%$ of RPSs and usually arise from major veins such as the IVC, gonadal veins or renal veins. They have a high risk of metastasis ( $50 \%$ at 5 years), but a lower risk of local recurrence $(10 \%$ at 5 years) $(4,6)$.

MPNSTs account for only $3 \%$ of RPS but are aggressive and high risk for both early local recurrence and distant metastases (4).

The wide variety of biological behaviour observed highlights the need for histological subtype to be taken into account by prognostic models, as a factor which contributes to recurrence risk.

Other tumour-related prognostic variables in RPS include grade, size and multifocality. Patient-related prognostic variables previously reported include age at presentation. Treatment-related variables comprise the occurrence of tumour rupture, surgical margins status and centre expertise $(6,7,9-18)$.

The prognostic variables listed above each contribute different amounts to the overall patient outcome and therefore need to be considered simultaneously in order to accurately predict risk.

Individualised prognosis prediction is of upmost importance in order to ensure adequate patient counselling and to inform clinical decision-making, especially regarding combination therapies.

In order to predict risk accurately, adequate staging systems and validated predictive tools are required.

This review critically appraises the current predictive tools available specifically for RPS and discusses their use within clinical practice.

\section{Staging systems for RPS}

The staging system used for patients with STS has recently undergone transformation. The 7 th edition of the American Joint Committee on Cancer/Union for International Cancer Control (AJCC/UICC) Staging Manual had limited ability to categorise RPS patients into meaningful prognostic groups, there were several reasons for this (19-24).

Firstly, all patients with STSs were categorized into stages according to tumour grade, size, depth, lymph node involvement and distant metastasis (25). Secondly, anatomical site or histological subtype were not considered as prognostic variables by the staging system. Finally, tumour size was subdivided into two categories; below $5 \mathrm{~cm}$ and above $5 \mathrm{~cm}$. Most RPSs are over $5 \mathrm{~cm}$ and frequently there is no lymph node involvement or distant metastasis. This meant that tumour grade was the only prognostic variable within the staging system relevant to the prognosis of RPS.

The staging system published in the $7^{\text {th }}$ edition Staging Manual had clear limitations, meaning its applicability to patients with RPS was limited at best. This was demonstrated in a study by Tan et al. (24). The authors applied the $7^{\text {th }}$ edition of the AJCC staging system to over 600 patients and obtained a low concordance index (0.62).

The recent $8^{\text {th }}$ edition of the AJCC Staging Manual was published with three major changes (26). Firstly, patients with STS were sub-categorized according to the anatomical site of the tumour (trunk and extremity, retroperitoneum, head and neck, abdomen and visceral organ system). Tumour size was also changed to incorporate four categories, instead of two previously (less than 5, 5-10, $10-15,15 \mathrm{~cm}$ and above).

In addition, the new staging system took into account the need for tools to predict individual prognosis and therefore incorporated Gronchi's nomograms (see Figures 1,2). These nomograms have enabled clinicians to predict the 7-year OS and disease-free survival (DFS) for primary resected RPS patients based on clinical variables. Unfortunately, there still isn't a validated predictive tool available for patients with recurrent or metastatic RPS (27).

\section{Nomograms}

Kattan et al. developed the first nomogram for STS in 2002 at the Memorial Sloan Kettering Centre, New York (28). This nomogram was designed to predict post-operative 12-year sarcoma specific death. Variables used in Kattan's nomogram were age at diagnosis, histology, size, site and depth.

Since the publication of Kattan's nomogram many other nomograms have been published. In recent years, there have been nomograms designed to predict outcomes for 


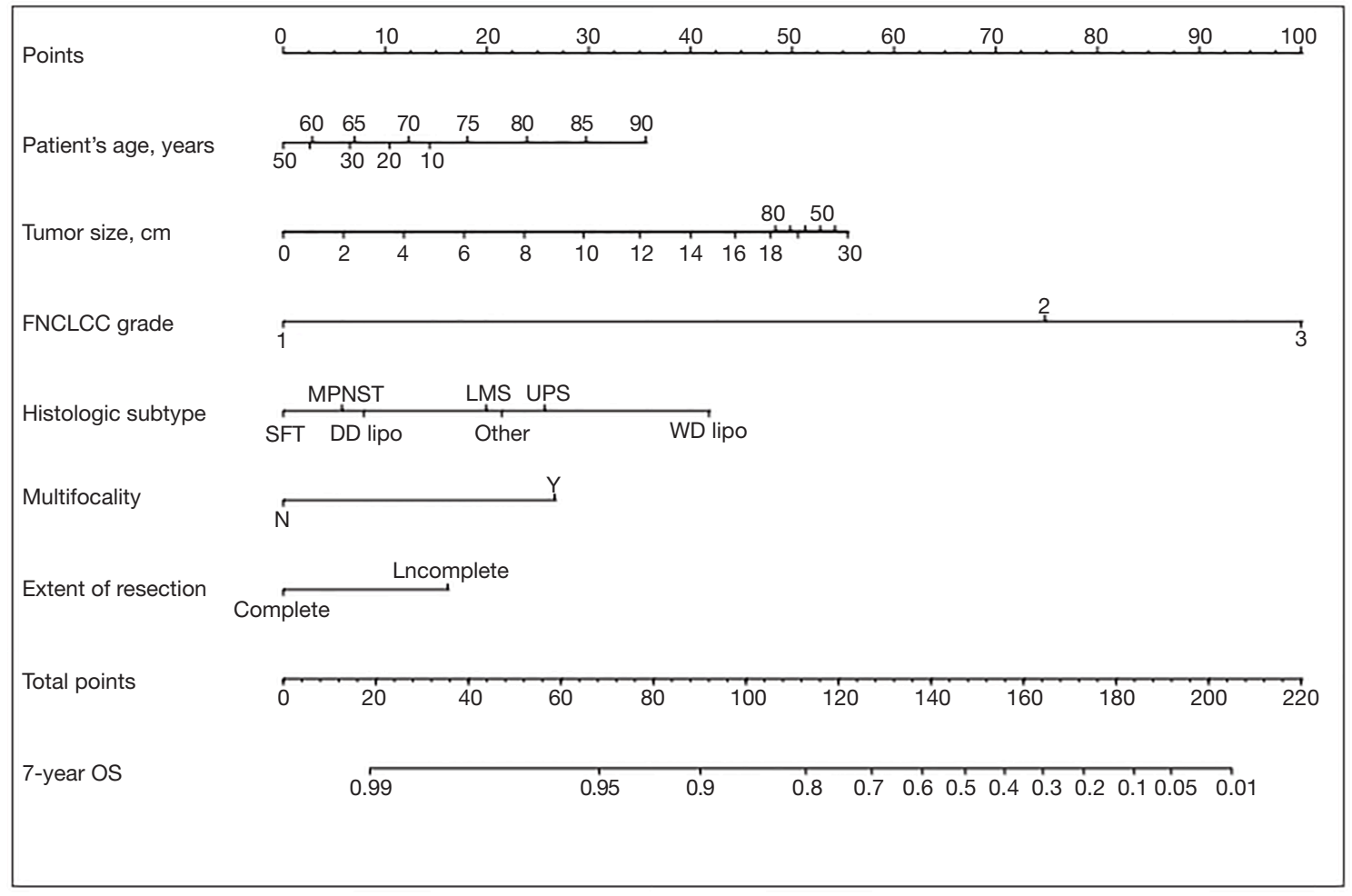

Figure 1 Gronchi's nomogram for predicting 7-year disease-free survival (DFS) in patients with primary resected retroperitoneal sarcoma. To use the nomogram, locate the patient's age and draw a vertical line up to the point axis, this will give you a points score for patient age. Repeat the above step for each of the covariates and add together the points scored for each covariate. Find the sum of the points on the total points axis and then draw a vertical line down to the axis labelled 7-year OS to find the predicted probability. Reprinted with permission from Gronchi et al. (27).

patients with specific histological subtypes of STS and also site-specific nomograms. Histology-specific nomograms are available for liposarcoma, uterine leiomyosarcoma, breast phyllodes tumours, synovial sarcoma, rhabdomyosarcoma and desmoid-type fibromatosis (29-36). There are also site-specific nomograms for retroperitoneal and extremity sarcomas $(21,22,24,27,37-42)$. Nomograms that are designed for use in specific patient subgroups allow the inclusion of variables that are particularly important in that definite setting.

\section{Rationale for RPS specific nomograms}

RPS is a rare disease, has great variability in its natural history and is associated with inferior outcomes when compared to extremity sarcoma. In RPS there are certain negative prognostic factors, such as multifocality, which are of particular importance but are rarely considered in extremity sarcoma. Also, it is difficult to consider RPS and extremity sarcoma as the same entity when considering variables such as tumour size and histological subtype, as RPSs are usually large at diagnosis and are limited to a few histological subtypes. This has obvious implications for the design of prognostic tools as different variables, and ranges within those variables, are required when comparing RPS and extremity sarcoma.

Due to the reasons discussed, nomograms, which have been developed to predict prognosis for all patients with STS have clear limitations. Table 1 presents published nomograms for RPS patients.

\section{Statistical considerations}

The aim of a prognostic nomogram, in cancer medicine, is to compute the probability of a specific outcome at certain time-points after definite treatment (surgery in the case of RPS).

The outcomes of interest can be OS (interval between surgery and death from any cause), DFS (interval between surgery and local recurrence, distant recurrence or death, 


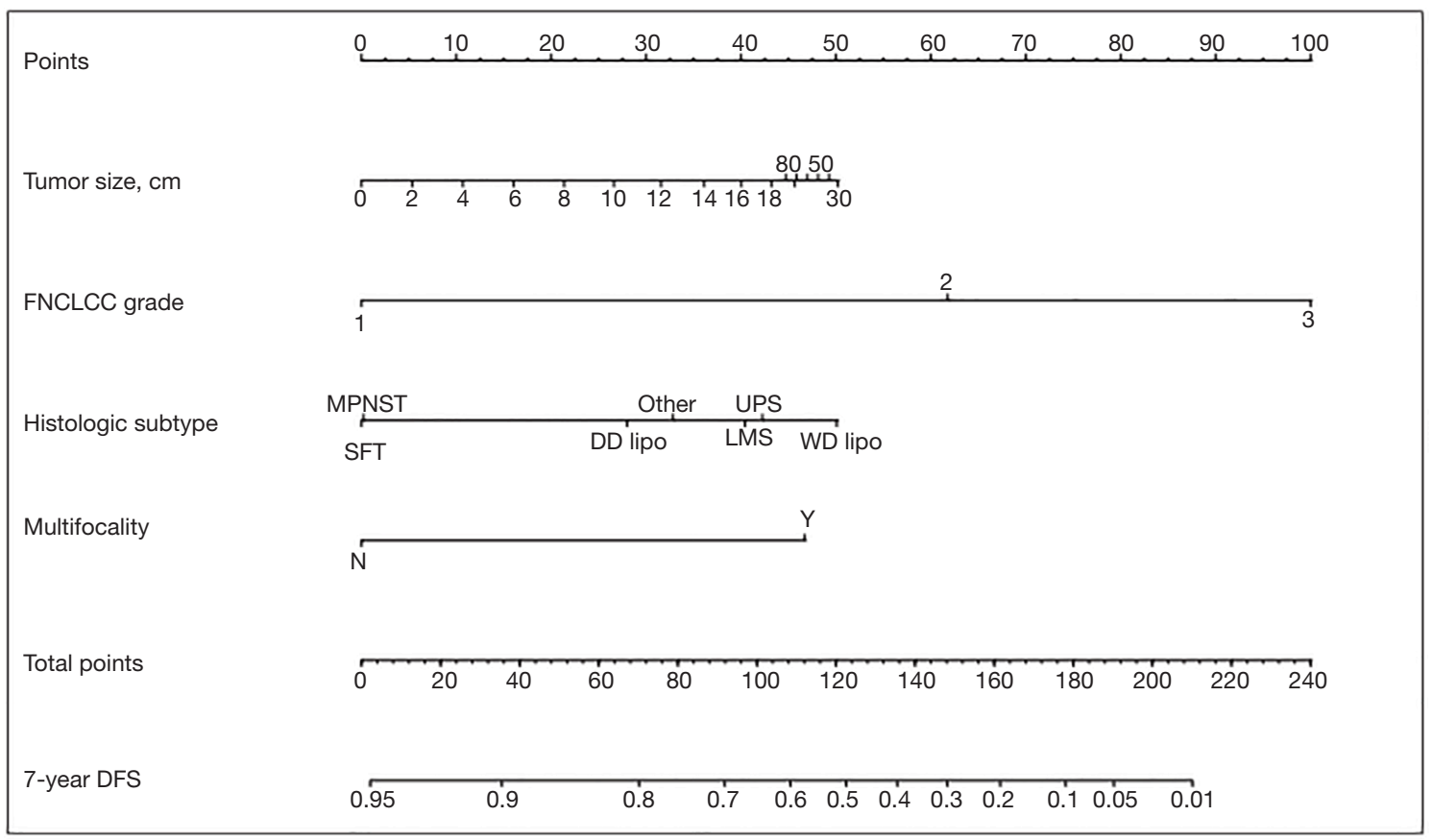

Figure 2 Gronchi's nomogram for predicting 7-year overall survival (OS) in patients with primary resected retroperitoneal sarcoma. To use the nomogram, locate the patient's tumour size and draw a vertical line up to the point axis, this will give you a points score for tumour size. Repeat the above step for each of the covariates and add together the points scored for each covariate. Find the sum of the points on the total points axis and then draw a vertical line down to the axis labelled 7-year DFS to find the predicted probability. Reprinted with permission from Gronchi et al. (27). DFS, disease-free survival.

whichever occurred first), crude cumulative incidence of local recurrence/distant metastasis or local recurrence/ distant metastasis free survival.

Nomograms are the graphical representation of a multivariable model in which the relative contribution of each covariate on the outcome of interest is factored. In nomograms, each covariate is depicted on an axis, its specific value is converted in a score and the sum of each score is converted into the outcome of interest.

When using a nomogram it is important to understand how far the predictions generated by the nomogram are from what is actually observed on average (calibration) and whether a nomogram is able to distinguish, between two patients, which of the two will develop the event of interest first (discrimination). Calibration is commonly assessed by means of calibration plots, in which the predicted values are plotted against the observed values. In a perfectly calibrated nomogram the plots would fall exactly on a 45-degree line. Discrimination is usually assessed with the concordance index (Harrell C-index) (43). The concordance index represents the probability that, among a randomly selected pair of patients with different outcomes, the nomogram would predict a worst prognosis for the patient who will develop the specified event first. The Harrell C-index can vary from 1 (perfect discrimination) to 0.5 (the chance of predicting the worst prognosis in the patient with the poorer outcome is completely random).

Both calibration and discrimination can be assessed by applying the nomogram to the same cohort of patients used to build the nomogram (internal validation), or to a different cohort (external validation). A nomogram can only be proven reliable in different cohorts by undergoing successful external validation.

Extensive reviews focusing on the statistical background of nomograms are published (44-46).

\section{Nomograms for RPS}

The inability of the $7^{\text {th }}$ edition AJCC staging system to stratify RPS patients into reliable prognostic groups and the limited ability of generic STS nomograms to accurately predict the prognosis of RPS patients, has led to the 


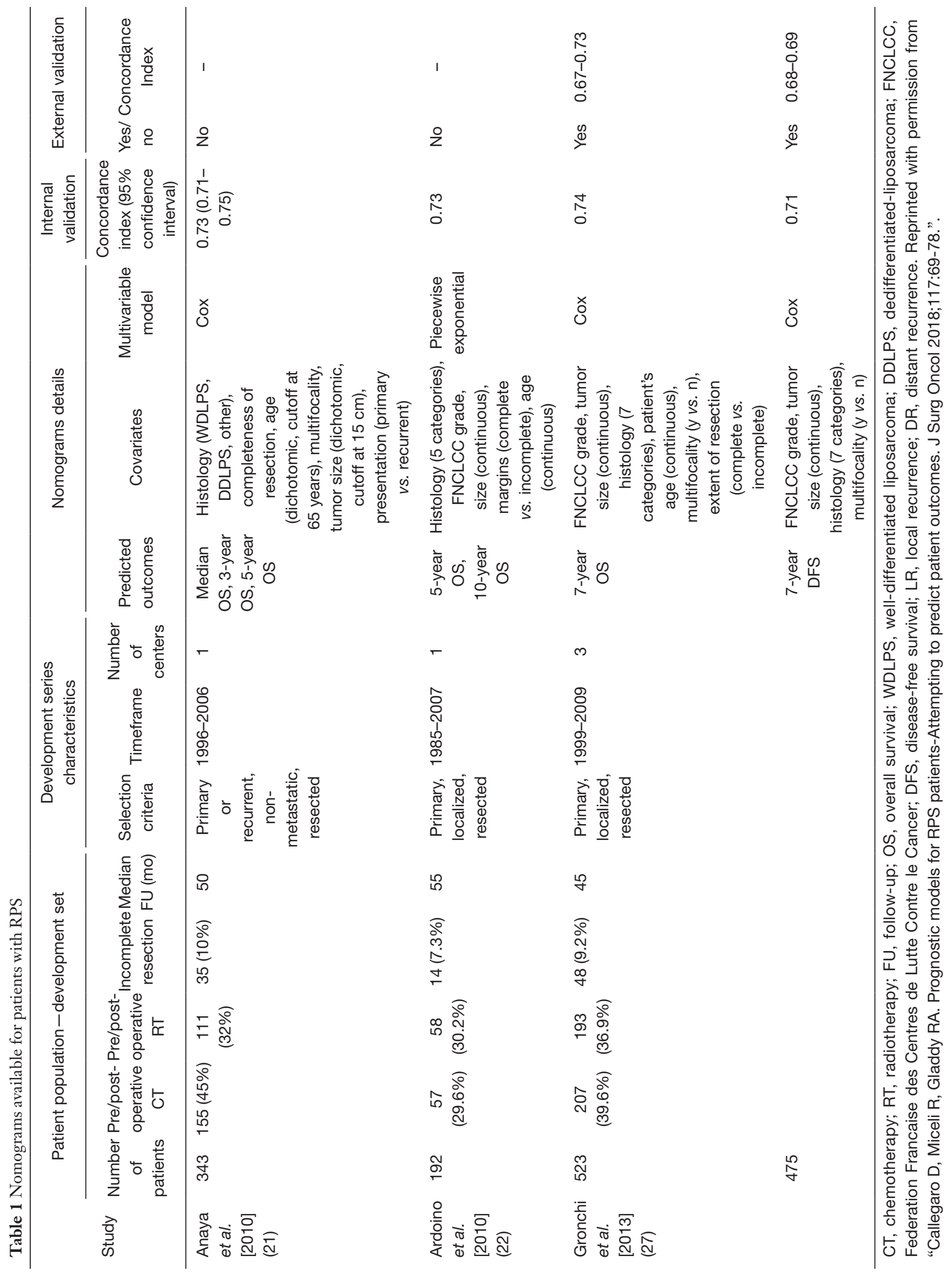


development of several RPS specific nomograms (24). The limited usefulness of Kattan's nomogram in RPS was demonstrated by Tan et al. who applied the nomogram to over 600 RPS patients and calculated the concordance index to be low (0.62) (24).

In 2006 Dalal et al. developed a nomogram for liposarcomas, unfortunately its utility as a predictive tool was limited in RPS patients as only $33.5 \%$ of patients in the developing set had RPS, with the majority of cases occurring in the extremities (29).

Anaya et al. developed the first RPS specific nomogram in 2010. It was designed for use in patients undergoing surgery with curative intent (21). Covariates included age (65 years cut off), tumour size ( $15 \mathrm{~cm}$ cut off), multifocality, completeness of resection, histological subtype and tumour presentation (primary or recurrent). Anaya's nomogram was derived using a multivariable Cox regression model and predicted median, 3- and 5-year survival. This was the first nomogram able to predict prognosis specifically for RPS patients, however it had several limitations. $30 \%$ of the developing cohort had recurrent tumours, which frequently behave differently due to biological and histological differences, potentially confounding the model. However, tumour presentation was considered as a variable and therefore should have captured this heterogeneity. It is worth noting that recurrent tumours of the retroperitoneum are predominately liposarcomas, which are associated with a poorer prognosis than that of primary tumours (47). Recurrent tumours may also be managed differently in terms of the extent of surgical resection, with surgery often aiming to increase the recurrence free interval rather than aiming for cure. Also age and tumour size had specified cut offs and weren't considered as continuous variables. Finally, the histology variable was only divided into 3 categories (WDLPS, DDLPS, other).

Also in 2010, Ardoino et al. developed a nomogram for RPS patients to predict 5- and 10-year OS. Covariates used were age, grade, histology, size and completeness of resection (22). Only patients with primary RPS were included and age at diagnosis and tumour size were managed as continuous variables. The use of continuous variables for tumour size identified that tumours up to $25 \mathrm{~cm}$ were associated with a worse prognosis, however beyond $25 \mathrm{~cm}$ risk actually decreased. This probably reflects that the majority of larger tumours are usually either WDLPS or intermediate grade DDLPS and have a more indolent course in comparison to high grade DDLPS or LMS. Another advantage of Ardoino's nomogram was the long observation period (10 years) allowing late recurrences common to certain histological subtypes to be observed. A limitation within this study was that WDLPS and DDLPS were classified as one entity when actually their biological behaviour has been shown to be very different. The nomogram was also constructed using only 192 patients.

Both of the nomograms proposed by Anaya et al. and Ardoino et al. only attempted to predict outcome in terms of OS and therefore don't predict the risk of local recurrence or metastasis. Neither of these nomograms underwent external validation so their applicability outside of the developing centres is unknown.

There was a turning point in predictive tools available for RPS patients in 2013 when Gronchi et al. published 2 externally validated nomograms, based on data from 3 major sarcoma centres (Istituto Nazionale dei Tumori, Milan, Italy; University of California Los Angeles, Los Angeles, CA; University of Texas MD Anderson Cancer Center, Houston, Texas) (27). The nomograms were created for patients with primary resected RPS and predicted both 7 -year OS and DFS. The development set comprised 523 patients. The use of data from multiple centres reduced any selection bias, which had been attributed to previous nomograms built using single institution data. Both nomograms were constructed using multivariable Cox regression models. Variables used in the OS nomogram included age, tumour grade, size, histology, multifocality and extent of surgical resection. The DFS nomogram did not include age or extent of surgical resection as variables. Unlike the nomogram produced by Anaya et al. age and tumour size were managed as continuous variables. The categories for histological subtypes were increased compared to previous nomograms and included seven different histological subtypes based on the most recent WHO Classification system (27). Both nomograms underwent three external validations and were shown to have good calibration and discrimination (Harrel C index ranging from $0.67-0.73$ ), even across different ethnic groups. Another advantage is that the data used to construct the nomograms is the most recent data used amongst all of the RPS specific nomograms. The significance of using recent data to build nomograms is that treatment strategies change over time. The patients included within this data will have undergone more current therapeutic strategies, such as more aggressive surgical resection to reduce the risk of local recurrence. Ten-year OS and DFS could not be predicted because of a lack of long-term patient follow up. Currently, 

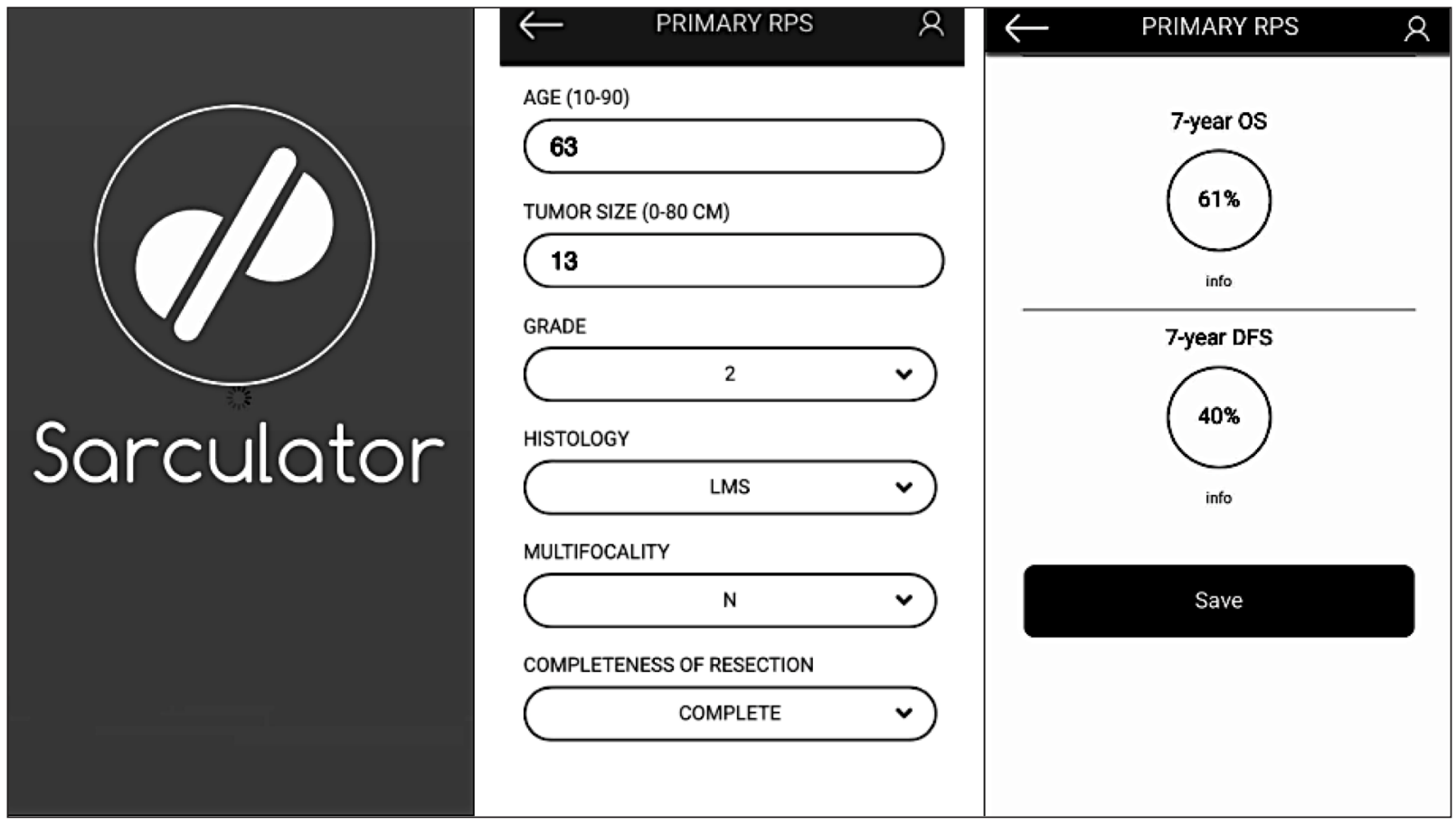

Figure 3 Screenshot from the Sarculator app. This example shows the app predicting 7-year DFS and OS for a 63 -year-old patient who has undergone complete primary resection of a $13-\mathrm{cm}$, grade 2 leiomyosarcoma that demonstrated no multifocality. The Sarculator app can be downloaded for free from official stores. DFS, disease-free survival; OS, overall survival.

the nomograms produced by Gronchi et al. are the most robust nomograms available for patients with primary resected RPS, hence there incorporation into the $8^{\text {th }}$ edition Cancer Staging Manual $(23,27,48)$. Gronchi's nomograms have been made available as a smart phone/tablet app named 'Sarculator', making it easy for clinicians to use predictive tools in day-to-day clinical practice (see Figure 3) (49).

There has been substantial effort by the international community to create robust prognostic tools for RPS. As you would expect, all of those currently published have advantages and limitations.

Gronchi's nomograms are currently the best available tools for predicting OS and DFS in patients with primary resected RPS, due to them being the only external validated tools available.

Prognostic tools for patients with recurrent RPS are limited, although Anaya's nomograms may be able to provide some prognostic information. However, due to the lack of external validation and because they are based on data from a single institution, they should be used with caution as their applicability outside of the developing institution is largely unknown.

\section{The use of RPS nomograms in clinical practice}

Nomograms allow for individualised prognosis prediction, they do not stratify patients into prognostic groups (the basis of the TNM staging system). For each individual, a point prediction can be derived from nomograms based on patient-, tumour- and treatment-related characteristics. Multivariable statistical models used to generate nomograms allow the association between variables to be taken into account so that an outcome can be generated.

Clinically, individualised prognostic tools are useful for counselling patients, assisting in therapeutic decisionmaking and in planning patient follow up. It is important to state that these predictive tools should not replace the use of clinical knowledge and judgement, but rather complement them.

Currently, the use of neoadjuvant/adjuvant chemotherapy (CT) in RPS is controversial. A meta-analysis of adjuvant CT in patients with localised STS showed only a small improvement in survival (HR 0.77, 95\% CI: 0.64-0.93, $\mathrm{P}=0.01$ ) (50,51). Similarly, the use of radiotherapy (RT) in RPS patients is unclear. There is little retrospective data 
about the role of RT in RPS, although it has been shown to have a limited benefit in the neoadjuvant setting due to technical issues and toxicity (52). The STRASS trial (NCT 01344018), a multicentre randomised prospective trial, is currently investigating the role of neoadjuvant RT in combination with primary surgical resection. The results of this trial will be available in a few years.

Currently available guidelines on the management of RPS highlight the uncertainty surrounding the use of CT and RT in patients with RPS. Consensus statements advocate multi-disciplinary case discussion to determine clinical strategies for each individual patient. Using predictive tools to estimate oncological risk at the time of surgery, may aid physicians in deciding which patients may benefit from adjuvant therapies.

It is important to consider that the prediction of outcome for each individual patient is a result of a statistical model. The prediction is based upon the mean outcome, in a hypothetical group of patients with a specific set of variables, it is also important to consider individual variability. This individual variability may be related to patient characteristics, such as comorbidities, that none of the currently available predictive tools for RPS take into account.

Another consideration is that as treatments change over time, nomograms can become out-dated and therefore require updates as guidelines change.

\section{The future of RPS prognosis prediction}

The development of robust, externally validated nomograms provides clinicians with a tool, which allows prediction of individual prognosis in RPS patients.

The $8^{\text {th }}$ edition of the AJCC Staging Manual incorporated Gronchi's nomograms to improve individual prognosis prediction in RPS patients. Currently available nomograms do not cover all disease stages so would not be able to replace the AJCC staging system. However, in the future it is possible that there will be nomograms for all disease stages and therefore a possibility that nomograms could eventually replace the AJCC staging system. At the current time, the AJCC staging system still provides clinicians with a simple way to stratify patients into prognostic groups.

Recruitment criteria for RCTs are mainly based on patient- or tumour-related characteristics such as tumour site or histological subtype. With the development of high quality nomograms it is possible that RCT inclusion criteria may shift to recruit patients on the basis of individual prognoses.

The construction of accurate nomograms requires data from high quality databases containing thousands of patients. In order to produce large patient databases in rare cancers such as RPS, international collaboration amongst sarcoma centres is vital. Transatlantic Retroperitoneal Sarcoma Working Group (TARPSWG, www.tarpswg. org) members are currently involved in a prospective observational study, known as 'RESAR' (Retroperitoneal Sarcoma Registry), which aims to build a high quality prospective database. The advantage of multicentre databases is that they avoid the inherent selection bias associated with data from single institutions. In the future, this database will provide the quality and volume of data required to build and validate robust and accurate nomograms for RPS patients.

Nomograms for patients with RPS mainly rely on postoperative variables, this means that their use is limited to prognosis prediction in post-operative patients. The implications of this, is that currently, nomograms have no role at the time of diagnosis or in unresectable disease. If a nomogram could be developed to predict prognosis in the pre-operative setting, it may be useful in determining which patients should receive neoadjuvant therapy.

Sadly, RPS is a disease whereby local recurrence and distant metastasis are not uncommon events. There is a great need for nomograms that predict prognosis in patients with recurrent or metastatic disease. All too often completed data sets for patients with recurrent or metastatic disease are hard to come by. This is often due to the patient not being treated for the primary tumour at a sarcoma referral centre, or because the physician changes throughout the treatment of the disease. Gathering sufficient data to create a nomogram for these patient groups requires collaboration between international cancer centres. TARPSWG presented nomograms specific for recurrent RPS at the 2017 Connective Tissue Oncology Society meeting, hopefully these will be available for use soon.

Nomograms available for RPS calculate a static prediction of the prognosis, usually post-operatively. A big disadvantage of currently available nomograms is that the prediction of prognosis is not updated according to follow up status or adjuvant treatment. The risk of metastasis in RPS falls a few years following surgery. The risk of local recurrence depends largely on tumour histology, although none of the nomograms available are able to demonstrate this change in risk over time. Variables shown to have a large influence shortly after the time of surgery may have 
less influence several years later. A nomogram with the ability to predict how risk changes with time and according to disease events would dramatically change individual prognosis prediction in RPS.

None of the predictive tools currently available for RPS take into account genomic, radiomic or immunological variables. Genomic markers have been shown to be associated with prognosis in STS (53). The inclusion of such variables may improve the ability of predictive tools to predict individual patient prognosis.

Finally, if nomograms are to be fully integrated into clinical practice, it is important that they are presented in a user-friendly format. Drawing lines on a nomogram is both inaccurate and time consuming. This can be overcome by using digital versions of the nomogram, these calculators can then be offered as a smart phone app or through a website. Offering prognosis prediction in such a way provides clinicians with a tool that can be integrated easily into clinical practice, for example in clinics when counselling patients and during multi-disciplinary team meetings. Last but not least they will make the selection of patients for clinical trials easier and more uniform.

\section{Acknowledgements}

None.

\section{Footnote}

Conflicts of Interest: The authors have no conflicts of interest to declare.

\section{References}

1. Singer S, Maki RG, O'Sullivan B. Soft tissue sarcoma. In: DeVita VT Jr, Lawrence TS, Rosenberg SA. editors. Cancer: Principles and Practice of Oncology: Primer of the Molecular Biology of Cancer. 9th ed. Philadelphia, PA: Lippincott Williams \& Wilkins, 2011.

2. Bonvalot $\mathrm{S}$, Raut CP, Pollock RE, et al. Technical considerations in surgery for retroperitoneal sarcomas: position paper from E-Surge, a master class in sarcoma surgery, and EORTC-STBSG. Ann Surg Oncol 2012;19:2981-91.

3. Bonvalot S, Miceli R, Berselli M, et al. Aggressive surgery in retroperitoneal soft tissue sarcoma carried out at highvolume centers is safe and is associated with improved local control. Ann Surg Oncol 2010;17:1507-14.
4. Gronchi A, Strauss DC, Miceli R, et al. Variability in patterns of recurrence after resection of primary retroperitoneal sarcoma (RPS): a report on 1007 patients from the multi-institutional collaborative RPS working group. Ann Surg 2016;263:1002-9.

5. Callegaro D, Fiore M, Gronchi A. Personalizing surgical margins in retroperitoneal sarcomas. Expert Rev Anticancer Ther 2015;15:553-67.

6. Gronchi A, Miceli R, Allard MA, et al. Personalizing the approach to retroperitoneal soft tissue sarcoma: histologyspecific patterns of failure and postrelapse outcome after primary extended resection. Ann Surg Oncol 2015;22:1447-54.

7. Toulmonde M, Bonvalot S, Meeus $\mathrm{P}$, et al. Retroperitoneal sarcomas: patterns of care at diagnosis, prognostic factors and focus on main histological subtypes: a multicenter analysis of the French Sarcoma Group. Ann Oncol 2014;25:735-42.

8. Pasquali S, Gronchi A, Strauss D, et al. Resectable extrapleural and extra-meningeal solitary fibrous tumours: a multi-centre prognostic study. Eur J Surg Oncol 2016;42:1064-70.

9. Stoeckle E, Coindre J, Bonvalot S, et al. Prognostic factors in retroperitoneal sarcoma. Cancer 2001;92:359-68.

10. Anaya DA, Lahat G, Liu J, et al. Multifocality in retroperitoneal sarcoma: a prognostic factor critical to surgical decision-making. Ann Surg 2009;249:137-42.

11. Keung EZ, Hornick JL, Bertagnolli MM, et al. Predictors of outcomes in patients with primary retroperitoneal dedifferentiated liposarcoma undergoing surgery. J Am Coll Surg 2014;218:206-17.

12. Anaya DA, Lev DC, Pollock RE. The role of surgical margin status in retroperitoneal sarcoma. J Surg Oncol 2008;98:607-10.

13. van Dalen T, Plooij JM, Van Coevorden F, et al. Longterm prognosis of primary retroperitoneal soft tissue sarcoma. Eur J Surg Oncol 2007;33:234-8.

14. Lewis JJ, Leung D, Woodruff JM, et al. Retroperitoneal soft-tissue sarcoma: analysis of 500 patients treated and followed at a single institution. Ann Surg 1998;228:355.

15. Gronchi A, Lo Vullo S, Fiore M, et al. Aggressive surgical policies in a retrospectively reviewed single-institution case series of retroperitoneal soft tissue sarcoma patients. J Clin Oncol 2009;27:24-30.

16. Bonvalot S, Rivoire M, Castaing M, et al. Primary retroperitoneal sarcomas: a multivariate analysis of surgical factors associated with local control. J Clin Oncol 2009;27:31-7. 
17. Strauss DC, Hayes AJ, Thway K, et al. Surgical management of primary retroperitoneal sarcoma. Br J Surg 2010;97:698-706.

18. Bremjit PJ, Jones RL, Chai X, et al. A contemporary large single-institution evaluation of resected retroperitoneal sarcoma. Ann Surg Oncol 2014;21:2150-8.

19. Nathan H, Raut CP, Thornton K, et al. Predictors of survival after resection of retroperitoneal sarcoma: a population-based analysis and critical appraisal of the AJCC staging system. Ann Surg 2009;250:970.

20. van Dalen T, Hennipman A, van Coevorden F, et al. Evaluation of a clinically applicable post-surgical classification system for primary retroperitoneal soft-tissue sarcoma. Ann Surg Oncol 2004;11:483-90.

21. Anaya DA, Lahat G, Wang X, et al. Postoperative nomogram for survival of patients with retroperitoneal sarcoma treated with curative intent. Ann Oncol 2010;21:397-402.

22. Ardoino I, Miceli R, Berselli M, et al. Histology-specific nomogram for primary retroperitoneal soft tissue sarcoma. Cancer 2010;116:2429-36.

23. Raut CP, Miceli R, Strauss DC, et al. External validation of a multi-institutional retroperitoneal sarcoma nomogram. Cancer 2016;122:1417-24.

24. Tan MC, Brennan MF, Kuk D, et al. Histology-based classification predicts pattern of recurrence and improves risk stratification in primary retroperitoneal sarcoma. Ann Surg 2016;263:593.

25. American Joint Committee on Cancer. AJCC cancer staging manual. 7th ed. New York: Springer, 2010.

26. American Joint Committee on Cancer. AJCC Cancer Staging Manual. 8th ed. Chicago: Springer, 2017.

27. Gronchi A, Miceli R, Shurell E, et al. Outcome prediction in primary resected retroperitoneal soft tissue sarcoma: histology-specific overall survival and disease-free survival nomograms built on major sarcoma center data sets. J Clin Oncol 2013;31:1649-55.

28. Kattan MW, Leung DH, Brennan MF. Postoperative nomogram for 12-year sarcoma-specific death. J Clin Oncol 2002;20:791-6.

29. Dalal KM, Kattan MW, Antonescu CR, et al. Subtype specific prognostic nomogram for patients with primary liposarcoma of the retroperitoneum, extremity, or trunk. Ann Surg 2006;244:381.

30. Zivanovic O, Jacks LM, Iasonos A, et al. A nomogram to predict postresection 5-year overall survival for patients with uterine leiomyosarcoma. Cancer 2012;118:660-9.

31. Tan PH, Jayabaskar T, Chuah K, et al. Phyllodes tumors of the breast: the role of pathologic parameters. Am J Clin Pathol 2005;123:529-40.

32. Canter RJ, Qin L, Maki RG, et al. A synovial sarcomaspecific preoperative nomogram supports a survival benefit to ifosfamide-based chemotherapy and improves risk stratification for patients. Clin Cancer Res 2008;14:8191-7.

33. Yang L, Takimoto T, Fujimoto J. Prognostic model for predicting overall survival in children and adolescents with rhabdomyosarcoma. BMC Cancer 2014;14:654.

34. Shen W, Sakamoto N, Yang L. Model to predict the survival benefit of radiation for patients with rhabdomyosarcoma after surgery: a population-based study. Int J Oncol 2014;45:549-57.

35. Chisholm JC, Marandet J, Rey A, et al. Prognostic factors after relapse in nonmetastatic rhabdomyosarcoma: a nomogram to better define patients who can be salvaged with further therapy. J Clin Oncol 2011;29:1319-25.

36. Crago AM, Denton B, Salas S, et al. A prognostic nomogram for prediction of recurrence in desmoid fibromatosis. Ann Surg 2013;258:347.

37. Callegaro D, Miceli R, Bonvalot S, et al. Development and external validation of two nomograms to predict overall survival and occurrence of distant metastases in adults after surgical resection of localised soft-tissue sarcomas of the extremities: a retrospective analysis. Lancet Oncol 2016;17:671-80.

38. Callegaro D, Miceli R, Mariani L, et al. Soft tissue sarcoma nomograms and their incorporation into practice. Cancer 2017;123:2802-20.

39. Cahlon O, Brennan MF, Jia X, et al. A postoperative nomogram for local recurrence risk in extremity soft tissue sarcomas after limb-sparing surgery without adjuvant radiation. Ann Surg 2012;255:343.

40. Mariani L, Miceli R, Kattan MW, et al. Validation and adaptation of a nomogram for predicting the survival of patients with extremity soft tissue sarcoma using a threegrade system. Cancer 2005;103:402-8.

41. Sampo M, Tarkkanen M, Tukiainen E, et al. A webbased prognostic tool for extremity and trunk wall soft tissue sarcomas and its external validation. Br J Cancer 2012;106:1076.

42. van Praag VM, Rueten-Budde AJ, Jeys LM, et al. A prediction model for treatment decisions in high-grade extremity soft-tissue sarcomas: Personalised sarcoma care (PERSARC). Eur J Cancer 2017;83:313-23.

43. Harrell FE, Lee KL, Mark DB. Multivariable prognostic models: issues in developing models, evaluating 
assumptions and adequacy, and measuring and reducing errors. Stat Med 1996;15:361-87.

44. Balachandran VP, Gonen M, Smith JJ, et al. Nomograms in oncology: more than meets the eye. Lancet Oncol 2015;16:e173-80.

45. Iasonos A, Schrag D, Raj GV, et al. How to build and interpret a nomogram for cancer prognosis. J Clin Oncol 2008;26:1364-70.

46. Collins GS, Reitsma JB, Altman DG, et al. Transparent reporting of a multivariable prediction model for individual prognosis or diagnosis (TRIPOD): the TRIPOD statement. BMC Med 2015;13:1.

47. Tseng WW, Madewell JE, Wei W, et al. Locoregional disease patterns in well-differentiated and dedifferentiated retroperitoneal liposarcoma: implications for the extent of resection? Ann Surg Oncol 2014;21:2136-43.

48. Chou YS, Liu CY, Chang YH, et al. Prognostic factors of primary resected retroperitoneal soft tissue sarcoma: analysis from a single Asian tertiary center and external validation of Gronchi's nomogram. J Surg Oncol 2016;113:355-60.

Cite this article as: Tattersall HL, Callegaro D, Ford SJ, Gronchi A. Staging, nomograms and other predictive tools in retroperitoneal soft tissue sarcoma. Chin Clin Oncol 2018;7(4):36. doi: 10.21037/cco.2018.08.01
49. DIGITAL FOREST. Sarculator. Available online: https://geo.itunes.apple.com/us/app/sarculator/ id1052119173? $\mathrm{mt}=8$

50. Pervaiz N, Colterjohn N, Farrokhyar F, et al. A systematic meta-analysis of randomized controlled trials of adjuvant chemotherapy for localized resectable soft-tissue sarcoma. Cancer 2008;113:573-81.

51. Gronchi A, Ferrari S, Quagliuolo V, et al. Histotypetailored neoadjuvant chemotherapy versus standard chemotherapy in patients with high-risk soft-tissue sarcomas (ISG-STS 1001): an international, open-label, randomised, controlled, phase 3, multicentre trial. Lancet Oncol 2017;18:812-22.

52. Tiong SS, Dickie C, Haas RL, et al. The role of radiotherapy in the management of localized soft tissue sarcomas. Cancer Biol Med 2016;13:373.

53. Chibon F, Lagarde P, Salas S, et al. Validated prediction of clinical outcome in sarcomas and multiple types of cancer on the basis of a gene expression signature related to genome complexity. Nat Med 2010;16:781. 